\title{
Remoção de corpo estranho orgânico após acidente motociclístico: Relato de caso incomum
}

\author{
Removal of organic foreign body after motorcycle accident: An unusual case report \\ Extracción de cuerpo extraño orgánico por accidente de motocicleta: Reporte de un caso inusual
}

Recebido: 05/05/2021 | Revisado: 10/05/2021 | Aceito: 21/06/2021 | Publicado: 18/07/2021

\author{
Ricardo Franklin Gondim \\ ORCID: https://orcid.org/0000-0002-3246-2299 \\ Hospital Instituto Doutor José Frota, Brasil \\ E-mail: rfranklingondim@gmail.com \\ Manoel de Jesus Rodrigues Mello \\ ORCID: https://orcid.org/0000-0001-6032-577X \\ Hospital Instituto Doutor José Frota, Brasil \\ E-mail: mjrmello@gmail.com \\ José Maria Sampaio Menezes Junior \\ ORCID: https://orcid.org/0000-0002-7218-3067 \\ Hospital Instituto Doutor José Frota, Brasil \\ E-mail: sampaiomenezes@gmail.com \\ Antônio Mont'Alverne Lopes Filho \\ ORCID: https://orcid.org/0000-0002-8963-9040 \\ Hospital Instituto Doutor José Frota, Brasil \\ E-mail: alverninho@yahoo.com.br \\ Jayara Ferreira de Aguiar \\ ORCID: https://orcid.org/0000-0002-6606-5849 \\ Hospital Batista Memorial, Brasil \\ E-mail: jayarafaguiar@hotmail.com \\ Josfran da Silva Ferreira Filho \\ ORCID: https://orcid.org/0000-0001-5391-3274 \\ Hospital Instituto Doutor José Frota, Brasil \\ E-mail: josfranctbmf@gmail.com \\ Thiago Rafael Silva Dantas \\ ORCID: https://orcid.org/0000-0002-9156-2222 \\ Hospital Instituto Doutor José Frota, Brasil \\ E-mail: thiagorafaeldantas94@gmail.com \\ Abrahão Cavalcante Gomes de Carvalho \\ ORCID: https://orcid.org/0000-0002-0581-2468 \\ Hospital Instituto Doutor José Frota, Brasil \\ E-mail: abrahao_cav@yahoo.com \\ Renato Luiz Maia Nogueira \\ ORCID: https://orcid.org/0000-0003-1940-0402 \\ Universidade Federal do Ceará, Brasil \\ E-mail: renatolmaia@terra.com.br
}

\begin{abstract}
Resumo
Introdução: Os traumas ao complexo orbitário são comuns durante acidentes e agressões, no entanto, a penetração de um corpo estranho de característica orgânica na cavidade orbitária é uma condição incomum quando comparada aos fatores etiológicos supracitados. Os ferimentos penetrantes na órbita podem ter repercussões clínicas mórbidas e em modo imediato e tardio no globo ocular e no sistema nervoso central. O objetivo do presente trabalho é relatar o diagnóstico e tratamento de um trauma incomum em complexo orbito-zigomatico-facial com abordagem multidisciplinar. Relato de caso: Paciente do sexo masculino, 22 anos de idade buscou o serviço de emergência do Hospital Instituto Doutor José Frota - CE (IJF) apresentando corpo estranho em região de cavidade orbitária esquerda após acidente motociclístico. Na tomografia computadorizada pré-operatória, observou-se: corpo estranho intraorbitário, no assoalho da órbita em trajeto obliquo súpero-inferior e látero-medial, transfixando o seio maxilar, com extremidade no espaço parafaríngeo e orofaríngeo direito Discussão: Durante o trauma na cavidade orbitária, $16 \%$ dos corpos estranhos permanecem na região e são classificados como corpos inorgânicos e orgânicos, sendo os orgânicos como relacionados a formações de abscessos intraorbitários em maior número do que o grupo divergente. Conclusão: O paciente evolui com 06 (seis) meses de acompanhamento pós-operatório, sem queixa álgica, estética ou funcional.
\end{abstract}

Palavras-chave: Corpo estranho; Órbita; Trauma maxilofacial. 


\begin{abstract}
Introduction: Trauma to the orbital complex is common during accidents and assaults, however, the penetration of a foreign body with an organic characteristic in the orbital cavity is an uncommon condition when compared to the aforementioned etiological factors. Penetrating orbiting wounds can have immediate and late morbid clinical repercussions on the eyeball and central nervous system. The aim of the present study is to report the treatment of complex and unusual trauma to the face. Case report: A 22-year-old male patient sought the emergency service of the Doctor José Frota Institute Hospital - CE (IJF) presenting a foreign body in the region of the left orbital cavity after a motorcycle accident. In the preoperative computed tomography, it was observed: intraorbital foreign body, with entry into the orbital floor and superior-inferior and latero-medial oblique path, transfixing the maxillary sinus, with extremity in the parapharyngeal and right oropharyngeal space Discussion: During the trauma in the orbital cavity, $16 \%$ of the foreign bodies remain in the region, they can be classified as inorganic (for example, glass and metal) and organic (for example, wood), and the group of organic foreign bodies represents a greater risk, due to the possible formation of intraorbital abscesses that develop during a continuous source of infection. Conclusion: The patient progresses with 06 (six) months of postoperative follow-up, without pain, aesthetic or functional complaints.
\end{abstract}

Keywords: Foreign body; Orbit; Maxillofacial trauma.

\title{
Resumen
}

Introducción: El traumatismo del complejo orbitario es común durante accidentes y agresiones, sin embargo, la penetración de un cuerpo extraño de característica orgánica en la cavidad orbitaria es una condición infrecuente en comparación con los factores etiológicos antes mencionados. Las heridas orbitales penetrantes pueden tener repercusiones clínicas mórbidas inmediatas y tardías en el globo ocular y el sistema nervioso central. El objetivo del presente estudio es informar sobre el tratamiento de traumatismos faciales complejos e inusuales. Caso clínico: Paciente varón de 22 años acudió al servicio de urgencias del Hospital Instituto Doutor José Frota - CE (IJF) presentando cuerpo extraño en región de cavidad orbitaria izquierda tras accidente de motocicleta. En la tomografía computarizada preoperatoria se observó: cuerpo extraño intraorbitario, con entrada al piso orbitario y trayecto oblicuo superior-inferior y latero-medial, traspasando el seno maxilar, con extremidad en el espacio parafaríngeo y orofaríngeo derecho Discusión: Durante el traumatismo En la cavidad orbitaria, el 16\% de los cuerpos extraños permanecen en la región, estos se pueden clasificar en inorgánicos (por ejemplo, vidrio y metal) y orgánicos (por ejemplo, madera), y el grupo de cuerpos extraños orgánicos representa un mayor riesgo., debido a la posible formación de abscesos intraorbitarios que se desarrollan durante una fuente continua de infección. Conclusión: El paciente evoluciona con 06 (seis) meses de seguimiento postoperatorio, sin dolor, molestias estéticas o funcionales.

Palabras clave: Cuerpo extraño; Orbita; Trauma maxilofacial.

\section{Introdução}

Os traumas de face são comuns durante acidentes automobilístico e agressões ao indivíduo, no entanto, a penetração de um corpo estranho de característica orgânica na cavidade orbitária é uma condição incomum quando comparada aos fatores etiológicos supracitados (Craft et al., 2011). Os ferimentos penetrantes na órbita podem ter repercussões clínicas mórbidas de modo imediato e tardio no globo ocular e no sistema nervoso central, ocasionando desde a uma pequena ferida em face ao óbito. A possibilidade da transfixação do objeto em complexo orbito-zigomático-maxilar até região intracraniana depende de fatores como: a energia do trauma, as características anatômicas do objeto (ponta e forma) e o ângulo formado entre o objeto penetrante e o crânio. A localização precisa do material retido sua relação com estruturas nobres craniofaciais como globo ocular, músculos extraoculares, nervo cranianos e encéfalo deve ser avaliada frente aos riscos e indicações ao procedimento cirúrgico e sua viabilidade. (Dell'avergana et al, 2016). Frente a traumas com as características citadas, a abordagem multidisciplinar entre as áreas de cirurgia e traumatologia bucomaxilofaciais, neurocirurgia e oftalmologia devem ser preconizadas com o intuito de acidentes e complicações neurológicas e oftalmológicas serem evitadas. Os exames imaginológicos são efetivos para auxiliar o diagnóstico, sendo a tomografia computadorizada considerada "padrão-ouro" devido a análise tridimensional e sem distorção em parâmetros métricos da extensão do objeto e sua relação com lesões óculocerebrais e fratura ósseas. (Das et al., 2020) 


\section{Metodologia}

O trabalho em questão aborda o caso de um paciente, de forma descritiva e qualitativa (Pereira,et al., 2018), portador de trauma maxilofacial, com o objetivo de detalhar o procedimento cirúrgico de remoção do projétil, sendo autorizado pelo paciente por meio de um Termo de Consentimento Livre e Esclarecido (TCLE) elaborado em linguagem fácil para obtenção do conhecimento acerca do destino das informações pessoais colhidas além dos riscos e benefícios para tal. O presente estudo tem por objetivo oferecer uma breve revisão da literatura, por meio da busca de artigos científicos e estudos de caso nas bases de dados MEDLINE, PubMed e Google Scholar, sobre os métodos de diagnóstico e planejamento de tratamento clínico para trauma por corpo estranho orgânico e sua reconstrução posterior.

\section{Relato de Caso}

Paciente do sexo masculino, 22 anos de idade, nega comorbidades e alergias ou uso contínuo de medicamentos. Buscou o serviço de emergência do Hospital Instituto Doutor José Frota - CE (IJF) apresentando corpo estranho em região de cavidade orbitária esquerda após acidente motociclístico com capacete, em zona rural do estado, um dia após o trauma. não sendo possível avaliar acuidade visual ou motilidade ocular ipsilateral; em olho direito apresentou acuidade visual e motilidade ocular preservada. Foi iniciado antibioticoterapia profilática com cefalotina 1 grama 06/06 horas endovenosa (EV), cetoprofeno 100mg 12/12 horas EV, dipirona 1G 06/06 horas EV e profilaxia antitetânica. Como exames complementares, foram solicitados: tomografia computadorizada de crânio e face (TC), radiografia póstero-anterior de tórax e hemograma completo, sendo detectável ao exame de imagem: Imagem hiperdensa compatível com corpo estranho intraorbitário com entrada no assoalho da órbita esquerdo e trajeto obliquo súpero-inferior e látero-medial, com transfixação em seio maxilar esquerdo e extremidade nos espaços parafaríngeo e orofaríngeo direito; fratura do assoalho orbitário e das paredes anterior e posterior do seio maxilar esquerdo, além de fratura do osso esfenóide e do etmoide ao longo do trajeto do corpo estranho. Ainda em análise, a presença de hemossinus maxilar, etmoidal e esfenoidal e obliteração da cavidade nasal à esquerda também foi visualizada; o corpo estranho apresenta-se comprimindo e deslocando superiormente o globo ocular esquerdo.

Figure 1: Figure 1-A e B: Aspecto clínico imediato do paciente em vista $3 / 4$ esquerda da face e vista em perfil esquerdo, respectivamente, evidenciando a presença de corpo estranho em região periorbitária esquerda com laceração corto-contusa em região periorbitária e oclusão ocular. Figure 1-C: laceração corto-contusa em região de orofaringe (parede posterior da faringe). Figure 1-D: Reconstrução tridimensional evidenciando corpo estranho com extensão desde a região periorbitária esquerda até região orofaríngea direita.

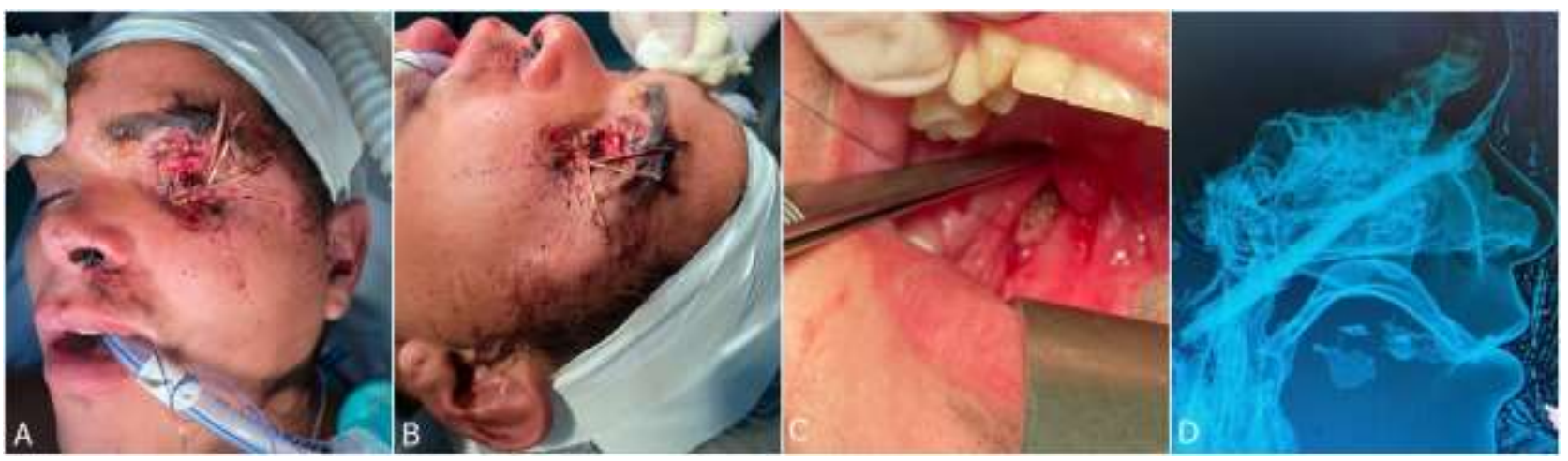

Fonte: Autores. 
O paciente foi submetido a centro cirúrgico de emergência para remoção de corpo estranho orgânico. $O$ fragmento foi removido com o auxílio de um fórceps 150 para apreensão adequada com uso de movimentos lentos e circulatórios com via de acesso a partir da laceração em região infraorbitária esquerda. Após remoção do corpo estranho, foi realizada irrigação abundante com soro fisiológico $0,9 \%$ e inspeção visual para realização de sutura por planos em laceração em região infraorbitária esquerda e parede posterior da faringe (orofaringe) com e fio vicryl 5- fio nylon 5-0 0. O paciente evoluiu com drenagem ativa de secreção serossanguinolenta três dias após a abordagem inicial, a qual justificou o uso de metronidazol 500mg EV 08/08 horas por 07 dias a antibioticoterapia, sanando o quadro infeccioso pós-operatório.

Figure 2: Aspecto clínico de fragmento removido da face do paciente.

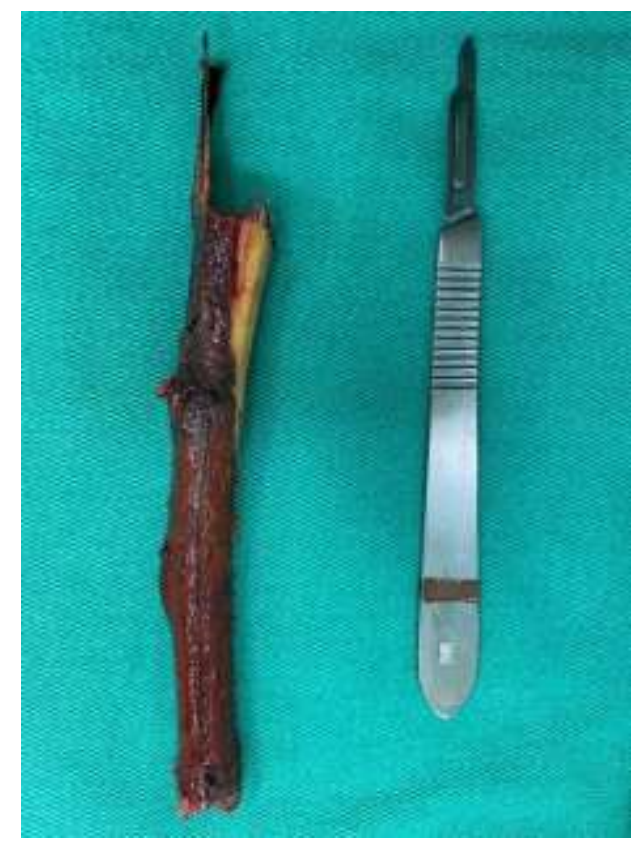

Fonte: Autores.

Após um mês da abordagem emergencial, o paciente apresentou: acuidade visual e motilidade ocular de ambos os olhos preservadas, ptose palpebral, distopia e enoftalmia do olho esquerdo. Ao exame de imagem, observou-se fratura de assoalho orbitário ipsilateral esquerdo 
Figure 3: Aspecto clínico pós-operatório de um mês, evidenciado motilidade ocular preservada apesar da fratura de assoalho presente
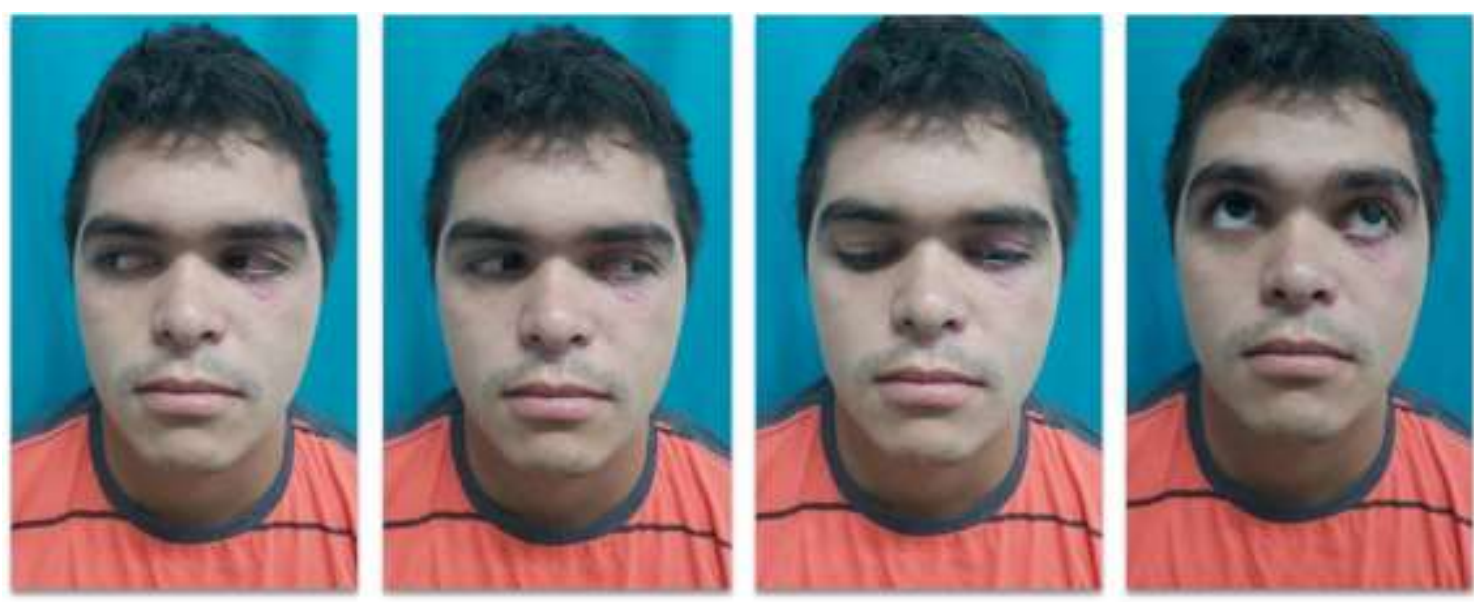

Fonte: Autores.

Após acompanhamento pós-operatório compatível com um mês de abordagem inicial de emergência, o paciente apresentava acuidade visual e motilidade ocular em ambos olhos, porém uma discreta distopia em olho esquerdo persistia, o que justificou a segunda abordagem cirúrgica para reconstrução orbitária, sendo realizada fixação de uma tela de titânio com parafusos do sistema 1.5mm no assoalho orbitário esquerdo. Paciente encontra-se em acompanhamento pós-operatório 6 meses após o trauma e evolui sem queixas álgicas, funcionais ou estéticas.

Figure 4: Figure 4-A: Reconstrução em 3D na TC de face e crânio após fixação de tela de titânio em assoalho orbitário esquerdo. Figure 4-B: Corte Axial da TC, o qual, a seta vermelha aponta a tela de titânio.
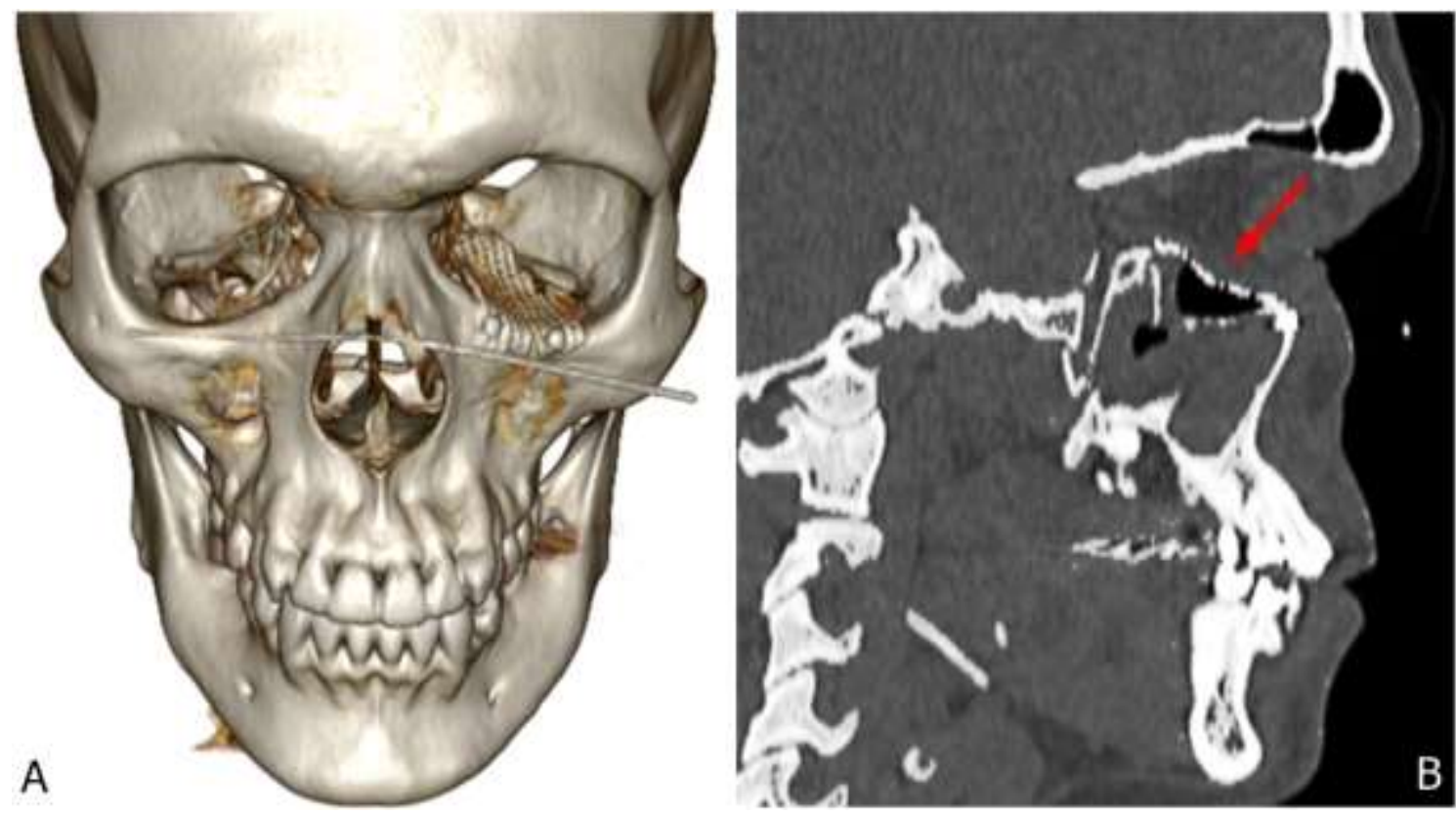

Fonte: Autores. 


\section{Discussão}

Devido a localização e projeção, o trauma na face ou no crânio pode estar associado a múltiplas lesões orbitais, podendo se caracterizar como fraturas orbitárias (assoalho orbitário, teto orbitário, parede medial, parede lateral, complexo zigomático-maxilar, complexo naso-orbito-etmoidal e ápice orbitário), lesões de tecidos moles, lesões as estrturas neurovasculares, músculos e envolver diretamente o globo ocular propriamente dito. Durante o trauma a cavidade orbitária, $16 \%$ dos corpos estranhos permanecem na região, classificados como corpos inorgânicos (vidro e metal) e orgânicos ( madeira), sendo o grupo de corpos estranhos orgânicos característico por apresentar risco maior ao indivíduo devido a formação de abscessos intraorbitários que se desenvolvem durante uma fonte contínua de infecção, pautados na reatividade inerente a natureza do material. (Escobar et al., 2016) (Weisman et al, 1983) (Oh et al., 2016). Os corpos estranhos orgânicos podem atuar favorecendo o crescimento bacteriano e sua retenção prolongada associada ao indivíduo pode ocasionar complicações como a celulite orbitária, o abscesso orbital e, em casos severos, a meningite; amaurose, oftalmoplegia persistente, ptose palpebral, diplopia binocular, distopia e paralisia dos movimentos oculares também são manifestações clínicas relacionadas ao corpo estranho retido em região craniofacial. O início de profilaxia antibiótica em corpos estranho inorgânicos é controverso, porém, é recomendado no grupo dos orgânicos. (Zoltán et al., 2020) (Sukegawa et al., 2016). Corpos estranhos de madeira apresentam baixa densidade ou até mesmo podem não ser notados na TC. Em imagens da TC de crânio e face, o coeficiente de absorção de corpos estranhos orgânicos (como a madeira) varia de -999 a +54 na escala de Hounsfield (HU). A TC padrão é realizada entre 200 HU e 350 e os corpos estranhos podem não ser identificados e descritos visto a sua densidade estar fora do espectro de hiperdensidade. A presença de ar intraorbital também dificulta a descrição e identificação da imagem, o que pode conduzir ao diagnóstico incorreto. (Zhao et al., 2019) (Li et al., 2016). A ressonância magnética é contraindicada quando há suspeita de fragmentos metálicos pelo risco de lesão ao paciente, além de demandar tempo maior para realização, o que é imprescindível no paciente politraumatizado que urge pronto-atendimento. O ultrassom de órbita pode ser considerado, no entanto, são proibidos quando há suspeitas de lesões do globo ocular. (Udhay et al., 2019) . A principal vantagem da TC no diagnóstico sobre os outros métodos de avaliação da órbita é a capacidade de identificar estruturas nobres anexadas a órbita, dentre elas, o nervo óptico; é importante averiguar a integridade dessas após traumas penetrantes, uma vez que o exame físico isolado não se pode confirmar a transecção do nervo óptico, por exemplo. Além disso, pode fornecer informações sobre lesões associadas dos seios paranasais e da cavidade craniana. (Weisman et al., 1983) Alguns objetos têm densidade diferente dos tecidos moles e duros da face e podem não ser distinguidos, aplicando-se principalmente aos corpos estranhos orgânicos, como a madeira. No presente caso, A TC sem contraste possibilitou a reconstrução em 3D, a qual tornou possível observar a extensão e dissecação do fragmento na face do paciente. Os corpos estranhos de madeira, devido aos poros em sua superfície e às características da matéria orgânica, facilitam o crescimento bacteriano e, se não forem removidos prontamente, são fatores etiológicos de infecções maxilofaciais em potencial e também são evidenciados como materiais de integridade frágil pós-trauma, tornando a sua remoção um procedimento mais complexo quando apresenta fragmentações, sendo necessário inspeção visual e exame imaginológico pós-operatório para garantir que todos os fragmentos foram removidos. (Li et al., 2016) O paciente relatado sofreu lacerações em pálpebra, região infraorbitária e em região posterior da faringe ocasionados pela cinética do trauma, com remoção realizada em menos de 24 horas após o trauma. A cirurgia tem como indicação o tamanho, a natureza e a localização do corpo estranho bem como a presença de outras lesões ou complicações relacionadas ao fragmento: compressão do nervo óptico, envolvimento dos músculos extraoculares e infecções. (Elyadari et al., 2018) É indicada também quando os seguintes sinais e sintomas se manifestam: sinais de infecção ou de inflamação persistente em região orbito-zigomático-maxilar, proptose, restrição dos movimentos oculares, quemose, compressão da região orbitária e músculos extraoculares e compressão de feixes vasculonervosos próximos a cavidade orbitária. A fratura orbitária associada a lesão por corpo estranho é observada em 64-78\% dos casos (Zoltán et al., 2020) e 
decisões sobre o momento para abordagem e complexidade cirúrgica são definidas pela condição sistêmica do paciente, com conduta cirúrgica e tratamento a ser realizado por uma equipe multidisciplinar. A remoção do fragmento de madeira foi realizada pela equipe de cirurgia bucomaxilofacial associada a cirurgia vascular, caso fosse necessário ligadura de vasos sanguíneos cervicais, visto a possibilidade de hemorragia profusa após a remoção do fragmento, o que não ocorreu.

\section{Conclusão}

A remoção de corpos estranhos orgânicos é um desafio para os cirurgiões devido a possibilidade de remanescentes na região ocasionados pela composição e deformação do fragmento durante o trauma e sua remoção. É importante ressaltar que o exame clínico inicial associado a exames complementares de imagem são fundamentais para a identificação e localização precisa dos corpos estranhos. O diagnóstico e a decisão cirúrgica muitas vezes necessitam de uma equipe multidisciplinar, sendo definido os profissionais levando em consideração a condição de cada paciente.

\section{Referências}

Craft, R. O., Eberlin, K. R., Stella, M. H., \& Caterson, E. J. (2011). Management of extensive maxillofacial trauma with bony foreign body within the orbit from a chainsaw injury. Eplasty, 11, e44.

Das, D. et al (2020). Large organic orbitocranial foreign body. BMJ Case Report. s13, 1-3, 2020.

Dell'Aversana, G., Marenzi, G., Piombino, P., Testa, D., De Riu, G., Abbate, V., Califano, L., \& Sammartino, G. (2016). Violence-related periorbital trauma with a retained foreign body: a case report. Journal of medical case reports, 10, 16. https://doi.org/10.1186/s13256-015-0779-1

Escobar Montatixe, D., Villacampa Aubá, J. M., Sánchez Barrueco, Á., Sobrino Guijarro, B., \& Cenjor Español, C. (2016). Transnasal, Transethmoidal Endoscopic Removal of a Foreign Body in the Medial Extraconal Orbital Space. Case reports in otolaryngology, 2016 , 1981456. https://doi.org/10.1155/2016/1981456

Elyadari, M. et al (2018). Orbital metallic foreign body: Case report. Journal Francais d'Ophtalmologie, 41, e205-e206,.

Fernández-Ferro, M., Fernández-Fernández, M., Fernández-Sanromán, J., Costas-López, A., \& López-Betancourt, A. (2019). Management of a Penetrating Orbital Trauma from an Unusual Foreign Body with Associated Eye Injury. Annals of maxillofacial surgery, 9(1), 214-217. https://doi.org/10.4103/ams.ams_25_19

Li, J. et al. (2016). Clinical diagnosis and treatment of intraorbital wooden foreign bodies. Chinese Journal of Traumatology - English Edition, 19, p. 322-325.

Marchioni, D., Bertossi, D., Soloperto, D., Bianconi, L., Procacci, P., \& Nocini, P. F. (2016). Traumatic Intraconal Foreign Body: Report of an Injury Corrected With Combined Surgical and Endoscopic Treatment. Operative neurosurgery (Hagerstown, Md.), 12(1), 14-18. https://doi.org/10.1227/NEU.0000000000001150

Mashriqi, F., Iwanaga, J., Loukas, M., D'Antoni, A. V., \& Tubbs, R. S. (2017). Penetrating Orbital Injuries: A Review. Cureus, 9(9), e1725. https://doi.org/10.7759/cureus. 1725

Oh, T. S., Jeong, W. S., Chang, T. J., Koh, K. S., \& Choi, J. W. (2016). Customized Orbital Wall Reconstruction Using Three-Dimensionally Printed Rapid Prototype Model in Patients With Orbital Wall Fracture. The Journal of craniofacial surgery, 27(8), 2020-2024. https://doi.org/10.1097/SCS.0000000000003195

Pereira A. S. et al. (2018). Metodologia da pesquisa científica. [free e-book]. Santa Maria/RS. Ed. UAB/NTE/UFSM. 5.3) Koche, J. C. (2011). Fundamentos de metodologia científica. Petrópolis: Vozes. Disponível em: http://www.brunovivas.com/wp content/uploads/sites/10/2018/07/K\%C3\%B6che-Jos\%C3\%A9Carlos0D0AFundamentos-de-metodologia-cient\%C3\%ADfica-_-teoria-da0D0Aci\%C3\%AAncia-e-inicia\%C3\%A7\%C3\%A3o-\%C3\%A0pesquisa.pdfht tps://repositorio.ufsm.br/bitstream/handle/1/15824/Lic_Computacao_Metodologia-Pesquisa-Cientifica.pdf?sequence=1

Sukegawa, S., Kanno, T., \& Furuki, Y. (2018). Application of computer-assisted navigation systems in oral and maxillofacial surgery. The Japanese dental science review, 54(3), 139-149. https://doi.org/10.1016/j.jdsr.2018.03.005

Weisman, R. A. et al (1983). Computed Tomography in Penetrating Wounds of the Orbit With Retained Foreign Bodies. Archives of Otolaryngology, 109, $265-268$.

Tomazic, P. V., Stammberger, H., Habermann, W., Gerstenberger, C., Braun, H., Gellner, V., Mokry, M., Klein, A., Langmann, G., \& Köele, W. (2011). Intraoperative medialization of medial rectus muscle as a new endoscopic technique for approaching intraconal lesions. American journal of rhinology \& allergy, 25(5), 363-367. https://doi.org/10.2500/ajra.2011.25.3663

Udhay, P., Bhattacharjee, K., Ananthnarayanan, P., \& Sundar, G. (2019). Computer-assisted navigation in orbitofacial surgery. Indian journal of ophthalmology, 67(7), 995-1003. https://doi.org/10.4103/ijo.IJO_807_18

Xue, R., Lai, Q., Sun, S., Lai, L., Tang, X., Ci, J., Zhang, Z., \& Wang, Y. (2019). Application of Three-Dimensional Printing Technology for Improved Orbital-Maxillary-Zygomatic Reconstruction. The Journal of craniofacial surgery, 30(2), e127-e131. https://doi.org/10.1097/SCS.0000000000005031 
Research, Society and Development, v. 10, n. 8, e8410815811, 2021

(CC BY 4.0) | ISSN 2525-3409 | DOI: http://dx.doi.org/10.33448/rsd-v10i8.15811

Zhao, Y., Liu, J., Wang, Z., Yan, Y., Han, J., \& Yang, D. (2019). Transnasal Endoscopic Retrieval of a Metallic Intraorbital Intraconal Foreign Body Facilitated by an Intraoperative Magnetic Stick. The Journal of craniofacial surgery, 30(7), e603-e605. https://doi.org/10.1097/SCS.0000000000005622

Zoltan, I. et al. The surgical removal of orbital foreign bodies: Double case report. Orvosi Hetilap, 161, 889-894, 2020. 